\title{
Occupational exposure to noise and the attributable burden of hearing difficulties in Great Britain
}

\author{
K T Palmer, M J Griffin, H E Syddall, A Davis, B Pannett, D Coggon
}

Occup Environ Med 2002;59:634-639

See end of article for authors' affiliations

Correspondence to: Dr K T Palmer, MRC Environmental

Epidemiology Unit, Southampton General

Hospital, Southampton

SO16 6YD, UK:

ktp@mrc.soton.ac.uk

Accepted 12 March 2002

\begin{abstract}
Aims: To determine the prevalence of self reported hearing difficulties and tinnitus in working aged people from the general population, and to estimate the risks from occupational exposure to noise and the number of attributable cases nationally.

Methods: A questionnaire was mailed to 22194 adults of working age selected at random from the age-sex registers of 34 British general practices (21 201 subjects) and from the central pay records of the British armed services (993 subjects). Information was collected on years of employment in a noisy job; and whether the respondent wore a hearing aid, had difficulty in hearing conversation, or had experienced persistent tinnitus over the past year. Associations of hearing difficulty and tinnitus with noise exposure were examined by logistic regression, with adjustment for age, sex, smoking habits, and frequent complaints of headaches, tiredness, or stress. The findings were expressed as prevalence ratios (PRs) with associated $95 \%$ confidence intervals $(\mathrm{Cls})$. Attributable numbers were calculated from the relevant PRs and an estimate of the prevalence of occupational exposure to noise nationally.

Results: Some $2 \%$ of subjects reported severe hearing difficulties (wearing a hearing aid or having great difficulty in both ears in hearing conversation in a quiet room). In men, the prevalence of this outcome rose steeply with age, from below $1 \%$ in those aged 16-24 years to 8\% in those aged 55-64. The pattern was similar in women, but severe hearing loss was only about half as prevalent in the oldest age band. Tinnitus was far more common in subjects with hearing difficulties. In both sexes, after adjustment for age, the risk of severe hearing difficulty and persistent tinnitus rose with years spent in a noisy job. In men older than 35 years with 10 or more years of exposure, the PR for severe hearing difficulty was $3.8(95 \% \mathrm{Cl} 2.4$ to 6.2$)$ and that for persistent tinnitus $2.6(95 \% \mathrm{Cl} 2.0$ to 3.4$)$ in comparison with those who had never had a noisy job. Nationally, some 153000 men and 26000 women aged 35-64 years were estimated to have severe hearing difficulties attributable to noise at work. For persistent tinnitus the corresponding numbers were 266000 and 84000 .

Conclusions: Significant hearing difficulties and tinnitus are quite common in men from the older working age range. Both are strongly associated with years spent in a noisy occupation-a predominantly male exposure. The national burden of hearing difficulties attributable to noise at work is sub-
\end{abstract} stantial.

\section{Main messages}

- The number of workers in Britain with hearing loss and tin nitus caused by occupational exposure to noise is not well established.

- In this survey, $2 \%$ of working aged adults reported severe hearing difficulties, the problem being greatest in middle aged men; tinnitus was even more common.

- Risk of severe hearing difficulty and persistent tinnitus rose with years spent in a noisy job.

- Nationally, we estimate that 153000 men and 26000 women aged 35-64 years have severe hearing difficulties attributable to noise at work.

- For persistent tinnitus, the corresponding numbers are 266000 men and 84000 women.
Estimates of the national burden of hearing impairment attributable to noise at work have varied widely, according to the source of information. In 1995, 14200 individuals were in receipt of state industrial injuries benefit for occupational deafness, ${ }^{3}$ but in a survey of self reported, work related illness, it was estimated that some 140000 people in Britain have deafness or tinnitus caused or made worse by their work, ${ }^{4}$ while in the period 1991-95, members of the Association of British Insurers handled some 230000 claims for noise related occupational deafness. ${ }^{5}$ More information is needed on the extent of hearing difficulties and tinnitus in working aged people, and on the attributable burden of disease arising nationally from occupational exposures to noise.

Abbreviations: $\mathrm{Cl}$, confidence interval; $\mathrm{NSH}$, National Survey of Hearing; PR, prevalence ratio

\section{Policy implications}

- The national burden of hearing difficulties attributable to noise at work is considerable.

- Work related tinnitus is also common, although often overlooked.

- Preventing these health outcomes is an important public health priority. 
Table 1 Hearing abilities and reported use of a hearing aid

\begin{tabular}{|c|c|c|c|c|}
\hline \multirow[b]{2}{*}{ Hearing difficulty in the right ear* } & \multicolumn{4}{|c|}{ Hearing difficulty in the left ear* (no. (\%† wearing hearing aid)) } \\
\hline & Slight or none & Moderate & $\begin{array}{l}\text { Severe or can't hear at } \\
\text { all }\end{array}$ & Missing \\
\hline Slight or none & $8854(0.4 \%)$ & $100(9.0 \%)$ & $42(26.8 \%)$ & $20(0.0 \%)$ \\
\hline Moderate & $88(12.6 \%)$ & $174(12.3 \%)$ & $31(48.4 \%)$ & $3(66.7 \%)$ \\
\hline Severe or can't hear at all & $52(13.5 \%)$ & $18(50.0 \%)$ & $67(64.2 \%)$ & $0(0.0 \%)$ \\
\hline Missing & $1051(0.1 \%)$ & $40(0.0 \%)$ & $22(5.9 \%)$ & $2345(0.1 \%)$ \\
\hline
\end{tabular}

*Difficulty in hearing a whispered voice nearby in a quiet room.

$\dagger \%$ of respondents - a few people did not answer the item on wearing of a hearing aid.

In 1997-98, a large postal survey was conducted to obtain national estimates of occupational exposure to vibration. ${ }^{67}$ Because of interest in the relation between exposure to sources of hand transmitted vibration and sensorineural hearing loss, ${ }^{8}$ the survey also collected information on exposure to noise and reported hearing difficulties, and this provided an opportunity to investigate further the impact of occupational noise exposure. The research forms the focus of a large governmental report, ${ }^{9}$ but in this paper we summarise our principal findings.

\section{METHODS}

The study sample comprised 21201 men and women, selected at random from the age-sex registers of 34 general practices across Britain, and 993 members of the armed services. The practices were chosen to give a broad coverage of Great Britain and to ensure that industries with known exposure to vibration were adequately represented in the sample. A questionnaire $^{6}$ was mailed to each subject, and a single reminder was sent about five weeks later to non-responders. To ensure that members of the armed forces (who are cared for by military rather than civilian doctors) were included, a smaller separate mailing was sent to a random sample of men and women identified from the central pay records of each service. Further details have been provided elsewhere. ${ }^{67}$

Among other things, the questionnaire asked about hearing difficulties, the wearing of a hearing aid, and tinnitus; current occupation; and the total number of years that had been spent working in noisy places where there was a need to shout to be heard, in five response categories-none, less than 12 months, $1-5$ years, $6-10$ years, and more than 10 years. (As an approxi- mate rule of thumb, the need to shout to be heard at arm's length suggests noise exposures liable to exceed 85-90 $\left.\mathrm{dB}(\mathrm{A}) \cdot{ }^{10}\right)$

Difficulty in hearing was ascertained by the question: "How well can you hear a person who is talking to you when he is sitting on your right [left] side in a quiet room?".

Responses were categorised for each ear according to a three point scale: (a) with no difficulty or only slight difficulty; (b) with moderate difficulty; and (c) with great difficulty or "cannot hear him at all". The question had been modelled on that used in the National Survey of Hearing, ${ }^{11}$ in which those who reported "moderate difficulty" in hearing conversation in a quiet room were found to have a mean hearing impairment of about $45 \mathrm{~dB}$ HL. Occupational deafness is commonly bilateral, and the level of handicap in individuals is strongly influenced by the hearing in their better ear; also, some subjects who wear a hearing aid may report their compensated hearing abilities. So, the hearing disability of individuals was subsequently classified as "severe" if they wore a hearing aid or reported severe hearing difficulty in both ears; as "none" if they reported no or only slight hearing difficulty in the better ear; and as "intermediate" in everyone else. In those who only reported on hearing in one ear, the category was determined according to the hearing ability of the ear for which information was provided.

Tinnitus was identified by the question: "During the past 12 months have you had noises in your head or ears (such as ringing, buzzing, or whistling) which lasted longer than five minutes?".

Tinnitus which was reported to occur most or all of the time was defined as "persistent tinnitus". ${ }^{12}$

Table 2 Prevalence of hearing difficulty and tinnitus by age and sex

\begin{tabular}{|c|c|c|c|c|c|c|}
\hline & \multicolumn{4}{|c|}{ Difficulty in hearing* } & & \\
\hline & \multicolumn{2}{|c|}{ Intermediate } & \multicolumn{2}{|c|}{ Severe } & \multicolumn{2}{|c|}{ Tinnitus most or all of the time } \\
\hline & No. & $\% \dagger$ & No. & $\% \dagger$ & No. & $\% \dagger$ \\
\hline \multicolumn{7}{|l|}{ Men } \\
\hline $16-24$ & 2 & $<1$ & 3 & $<1$ & 9 & 1 \\
\hline $25-34$ & 6 & $<1$ & 9 & 1 & 32 & 2 \\
\hline $35-44$ & 34 & 3 & 11 & 1 & 61 & 4 \\
\hline $45-54$ & 49 & 4 & 32 & 2 & 104 & 7 \\
\hline $55-64 \ddagger$ & 61 & 6 & 78 & 8 & 154 & 13 \\
\hline All & 152 & 3 & 133 & 2 & 360 & 6 \\
\hline \multicolumn{7}{|l|}{ Women } \\
\hline $16-24$ & 1 & $<1$ & 5 & 1 & 16 & 2 \\
\hline $25-34$ & 8 & 1 & 8 & 1 & 18 & 1 \\
\hline $35-44$ & 10 & 1 & 11 & 1 & 35 & 3 \\
\hline $45-54$ & 12 & 1 & 25 & 3 & 49 & 4 \\
\hline $55-64 \ddagger$ & 23 & 3 & 28 & 4 & 49 & 5 \\
\hline All & 54 & 1 & 77 & 2 & 167 & 3 \\
\hline
\end{tabular}

${ }^{*}$ Categories as defined in text

†Denominators were all subjects in the relevant age bands with non-missing data for the variable in question.

łncludes a few men and women who were 64 when the mailing lists were assembled but 65 when the questionnaire was returned. 


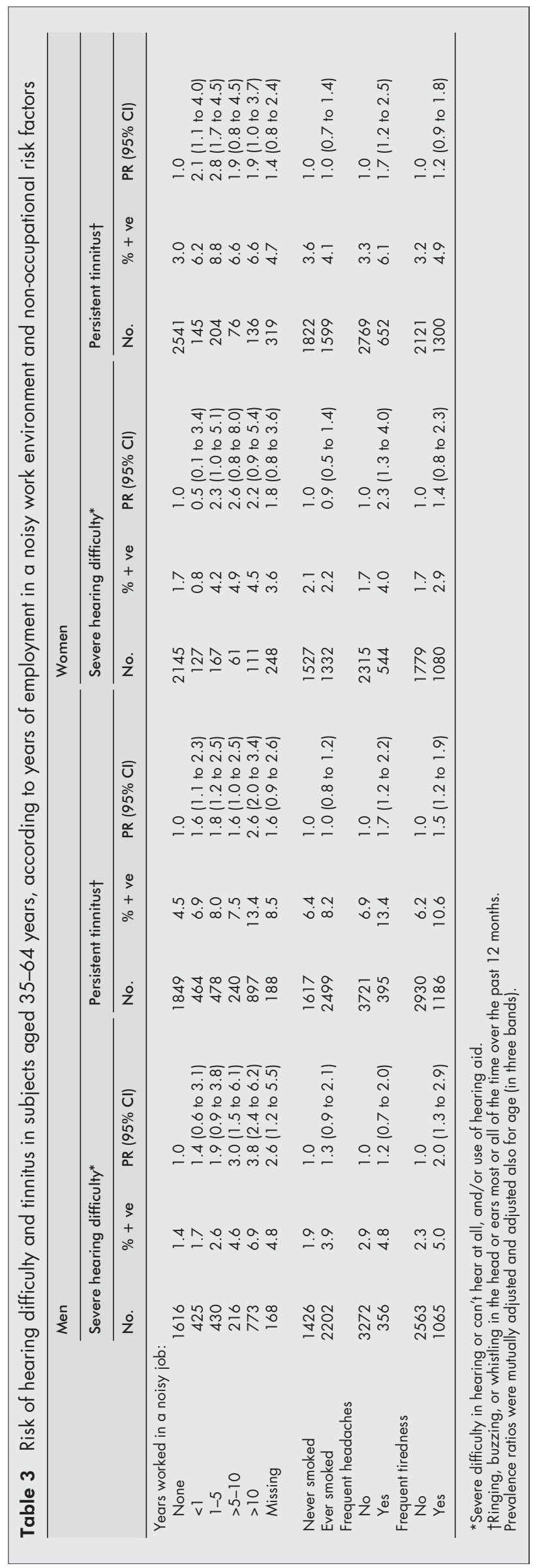

Information was also collected on age, sex, and smoking habits, as potential confounding factors, ${ }^{13}{ }^{14}$ and on complaints of frequent headaches, and frequently feeling tired or stressed, as an index of a generally lower threshold for reporting symptoms (a possible source of bias). All statistical analyses were carried out using STATA, release $5 .{ }^{15}$ Associations of hearing impairment and tinnitus with noise exposure were examined by logistic regression, with adjustment for age, sex, and the other factors. The findings were expressed as prevalence ratios (PRs) with associated 95\% confidence intervals (CIs), which were derived from the corresponding odds ratios according to a formula proposed by Zocchetti and colleagues. ${ }^{16}$ Attributable proportions (the proportions of cases of hearing impairment that would be avoided if the excess prevalence associated with exposure to noise were eliminated) and attributable numbers of cases were then calculated from the relevant PRs and from estimates of the prevalence of exposure to noise at work and the number of cases with hearing impairment nationally. The last two parameters were derived from prevalence estimates in the study sample, standardised according to the age-sex distribution of Great Britain in the most recent national census $^{17}$ (as described in an earlier report ${ }^{7}$ ). This part of the analysis excluded members of the armed forces (among whom deafness was uncommon). Confidence intervals for the associated attributable numbers were calculated by a bootstrap method. ${ }^{18}$

\section{RESULTS}

Questionnaires were completed by 12907 (58\%) of the sample selected for study. Among the non-responders were 221 (1\%) whom general practitioners advised us not to approach, and $1028(4.6 \%)$ who had moved away or died. The occupational distribution of responders was similar to that in the national census except that the armed forces were somewhat over represented. Non-response was more common in the younger age bands and in men. Further details of the pattern of response are reported elsewhere.

Table 1 records the level of hearing difficulty reported for each ear, and the prevalence of wearing a hearing aid. Among those providing full details, 9136 subjects had slight or no difficulty in the better ear, 67 reported severe bilateral difficulties in hearing, and 223 reported difficulties intermediate between these. Information on hearing abilities was missing for 1113 subjects in respect of the right ear, and for 23 subjects in respect of the left ear (suggesting a strong order effect in completing the questionnaire).

Altogether, 165 respondents reported that they wore a hearing aid-the prevalence varying among those who answered fully from $0.4 \%$ in those with slight or no difficulties in both ears to $64 \%$ in those reporting severe bilateral hearing difficulties. The prevalence rose with increasing difficulties in each ear, but was low for those with missing information, and only $0.1 \%$ of the subjects who had omitted both of the questions on hearing ability said that they wore a hearing aid.

After categorising subjects with partial information according to the hearing ability of the ear for which information was provided, and after classifying those who wore a hearing aid as severely affected, 210 subjects were counted as having severe hearing difficulties, 10001 as having no hearing difficulty, and 206 as having intermediate hearing difficulty. A further 2490 subjects failed to report on hearing in either ear or use of a hearing aid.

The prevalences of hearing difficulties and tinnitus were strongly related to age, severe difficulties in hearing being unusual under the age 35 of years (table 2). Thus, fewer than $1 \%$ of young men (aged 16-24) reported severe difficulties in hearing, compared with $8 \%$ of older men (aged 55-64). A similar age related trend was found for intermediate levels of hearing difficulty in men, for both categories of hearing difficulty in women, and for persistent tinnitus in both sexes. In 
Table 4 Estimated number of cases of hearing difficulty and tinnitus in adults aged 35-64 years attributable to occupational noise exposure in Great Britain

\begin{tabular}{|c|c|c|c|}
\hline & Severe hearing difficulty $\dagger$ & Persistent tinnitus $\ddagger$ & Either or both \\
\hline \multicolumn{4}{|l|}{ Men } \\
\hline PR (ever $v$ never employed in a noisy job)* & 2.9 & 2.1 & 2.3 \\
\hline Attributable proportion & $50.5 \%$ & $36.0 \%$ & $39.8 \%$ \\
\hline Attributable no. in Great Britain & 153000 & 266300 & 387400 \\
\hline$(95 \% \mathrm{CI})$ & $(88600-217300)$ & $(175600-357000)$ & $(277100-497700)$ \\
\hline \multicolumn{4}{|l|}{ Women } \\
\hline PR (ever $v$ never employed in a noisy job) * & 1.8 & 2.5 & 2.2 \\
\hline Attributable proportion & $12.4 \%$ & $20.9 \%$ & $17.9 \%$ \\
\hline Attributable no. in Great Britain & 25800 & 84000 & 97200 \\
\hline$(95 \% \mathrm{CI})$ & $(0-56000)$ & $(37900-130200)$ & $(47500-147000)$ \\
\hline
\end{tabular}

*Ever worked in a noisy place where there was a need to shout to be heard.

† Severe difficulty in hearing or can't hear at all, or use of hearing aid, or both.

$\ddagger$ Ringing, buzzing, or whistling in the head or ears most or all of the time over the past 12 months.

The PRs were adjusted for age (in three bands), tiredness or stress, frequent headaches, and smoking habits. The prevalence of exposure and the number

of cases in the population were estimated after standardising the sample estimates according to the 1991 census estimate of the age distribution of men

and women nationally. Estimates have been rounded to the nearest hundred. Bootstrapped Cls for the attributable numbers were based on 200

resampling iterations.

comparison with men, however, severe hearing loss was only around half as prevalent among women from the oldest age band, and persistent tinnitus was also less common (3\% v6\% overall and 5\% v 13\% in those aged 55-64 years).

Self reports of tinnitus and hearing difficulties were strongly associated. Thus, among men, the age standardised prevalence of persistent tinnitus was $16.1 \%$ in those who reported severe difficulties in hearing, compared with $5 \%$ in those with slight or no difficulties of hearing in the better ear; in women, the corresponding figures were $33.1 \%$ and $2.6 \%$.

Men more often reported having experienced prolonged exposure to noise at work. Around a third of men and $11 \%$ of women had worked in a noisy job for a year or longer, with $16 \%$ of men and $3 \%$ of women reporting more than 10 years of such exposure. Long term exposure was uncommon below the age of 35 years, but in the 55-64 year age band, $26 \%$ of men and $5 \%$ of women reported such exposures.

In view of the low prevalence of hearing loss at younger ages and the shorter duration of noise exposure, subsequent analyses focused on men and women aged 35-64 years. Table 3 shows the risks of severe hearing difficulty and persistent tinnitus in this group according to years worked in a noisy job, and their association with smoking habits, complaints of frequent tiredness or stress, and frequent headaches.

Smoking habits showed no clear relation with hearing loss or tinnitus. There were, however, significant associations with report of frequent headaches (PR 1.7 for tinnitus in men, and 1.7 to 2.3 for severe hearing difficulties and tinnitus in women), and of tiredness or stress (PRs 1.5 to 2.0 for the two outcomes in men).

After allowance for these factors and for age, the risk of auditory symptoms rose with years of employment in a noisy job. In men with 10 or more years of exposure, the PR for severe hearing difficulty was 3.8 (95\% CI 2.4 to 6.2 ) and that for persistent tinnitus 2.6 (95\% CI 2.0 to 3.4) in comparison with those who had never had occupational exposure to noise. Among women, those who reported occupational exposure to noise more often had severe hearing difficulty and persistent tinnitus (with PRs of around 2), but the relation to duration of noise exposure was less clear cut. An increased risk of severe hearing difficulty was also found in 168 men who did not complete the question on lifetime occupational exposure to noise (PR 2.6, 95\% CI 1.2 to 5.5 ).

Table 4 provides estimates of the number of men and women aged 35-64 years with severe hearing difficulty and persistent tinnitus attributable to occupational noise exposure nationally, based on a dichotomous classification of exposure (ever versus never worked in noisy places where there was a need to shout to be heard). Altogether, it was estimated that some 153000 men and 26000 women in this age band had attributable severe hearing difficulties; 266000 men and 84000 women were estimated to have attributable persistent tinnitus; and some 387000 men and 97000 women to have either or both types of complaint.

\section{DISCUSSION}

Our findings indicate that hearing difficulty and tinnitus both increase with age and tend, among those of working age, to be to a particular problem in men approaching retirement. The data also confirm that the prevalence of hearing loss and tinnitus increases with duration of occupational exposure to noise, even after allowance for age; and indicate that occupational noise exposure is responsible for an estimated 153000 men and 26000 women with severe hearing difficulties in the 35-64 year age band in Great Britain, as well as a considerable burden of disease from persistent tinnitus.

A particular strength of our survey was the large sampling base which included over 22000 randomly selected subjects. Almost everyone in Great Britain registers with a general practitioner except those in the Armed Forces who were sampled separately, and so the initial sample is likely to have been adequately representative of working aged adults in Britain. Practices were selected to ensure that industries with known exposure to vibration were adequately represented in the sample, but a broad geographical coverage was achieved and the distribution of occupations in respondents was similar to that at the last national census, providing a reasonable basis for estimating the national burden of hearing loss attributable to noise.

The response rate was $58 \%$, and the prevalence of hearing impairment and tinnitus could have been overestimated if those with auditory symptoms returned a questionnaire preferentially. However, the questionnaire did not focus on hearing impairment or noise exposure specifically, the distribution of occupations in respondents was representative of Great Britain as a whole, and the prevalence of auditory symptoms was similar in those who responded with and without a reminder (whereas response bias might lead to a higher prevalence in early than late responders). The questionnaire mainly concerned vibration, which is a well recognised source of noise exposure, and so a higher response rate among users of vibratory tools might also have biased the estimate of hearing loss, especially in men; but the prevalence of exposure to hand transmitted vibration was independently corroborated by a second community survey, ${ }^{2}$ and again varied little according whether a reminder was required. ${ }^{7}$ These observations argue against a major response bias. 
Errors might also arise from inaccuracies in the information supplied by subjects. Thus, the association of noise exposure with hearing loss and tinnitus could have been exaggerated if those with hearing problems recalled their noise exposure history more completely, or if those with a long history of noise exposure paid more attention to their hearing difficulties. Conversely, non-differential errors in reporting of exposure or auditory symptoms would bias risk estimates towards the null value. However, the validity of the questions on hearing impairment and tinnitus have been previously established,"112 and major error from this source seems unlikely.

A significant minority of subjects failed to report on their hearing abilities, raising the possibility that the attributable burden of hearing loss at the ages studied was underestimated. This seems unlikely, however, as the prevalence of using a hearing aid was similar in these subjects to that in subjects who reported good levels of hearing ability.

Finally, our measure of exposure to noise (lifetime years in a job where there was a need to shout to be heard) was necessarily fairly crude, and did not include an index of intensity or allow estimation of lifetime Leq. This may have resulted in some misclassification between exposure bands in table 3 , and may have weakened our estimates of risk.

There have been several previous investigations of hearing impairment in Britain. ${ }^{11}{ }^{1920}$ In the most comprehensive of these, the National Survey of Hearing (NSH), ${ }^{11}$ over 48000 questionnaires were mailed to randomly selected subjects from four British cities. The questions on hearing difficulties were similar to those used in the current study. The prevalence of severe hearing difficulty (great difficulty or inability to hear at all in the better ear) ranged from $0.2 \%$ in $17-30$ year olds to $4.1 \%$ in those aged $61-70$ years. More than $3 \%$ of respondents reported at least moderate bilateral difficulty in hearing in the quiet. A subset underwent comprehensive audiological assessment, among whom, in the 51-60 year age band, $8 \%$ had bilateral hearing impairment of 35-45 dB HL and 5\% had a greater deficit.

In the $\mathrm{NSH}^{11}$ and in a six city Italian study using a similar study design, ${ }^{21}$ hearing impairment was found to be about twice as common in manual as in non-manual workers. Associations were found in the general population between hearing loss and occupational noise exposure: in the NSH, an estimated exposure at more than $90 \mathrm{~dB}(\mathrm{~A})$ Leq for a 50 year working lifetime increased the odds of $\geqslant 45 \mathrm{dBHL}$ in the better ear by 2.3 -fold after adjustment for age and sex; while in the Italian study noise exposure (at an undefined level) increased the odds of a loss $\geqslant 25 \mathrm{dBHL}$ by 2.1 -fold and a loss $\geqslant 45 \mathrm{dBHL}$ by 1.7 -fold. These findings are broadly compatible with our own.

Tinnitus has also been widely surveyed in the community, and its prevalence has varied considerably according to case definition. Thus, in one British survey, $21-39 \%$ of adults had ever experienced "any noises" in their ears or head ${ }^{19}$; while, in an OPCS study, $22 \%$ said that they had heard noises in their head or ears such as ringing or buzzing sounds, although in a third of these the noises were brought on only by the stimulus of a loud noise, water in the ears or catarrhal illness, and only $2 \%$ were "bothered a great deal" by them..$^{22}$ In an analysis based on 6804 subjects from the NSH, the point prevalence of tinnitus which was spontaneous (not immediately following noise) and more than transient (lasting at least five minutes) varied little (from $15.5 \%$ to $18.6 \%$ ) between the four cities surveyed, but the prevalence of severely annoying symptoms varied more widely (from $0.4 \%$ in Nottingham to $2.8 \%$ in Glasgow) $)^{12}$; and in a later more detailed phase of the NSH, persistent spontaneous tinnitus was reported in $9.7 \%$ of all adults, with a prevalence of $5.7 \%$ in $17-30$ year olds and $12.4 \%$ in $51-60$ year olds. ${ }^{11}$

Several investigators have found an increased prevalence of tinnitus in manual workers, ${ }^{21}{ }^{23}$ and in workers with occupa- tional exposure to noise. ${ }^{23}$ Coles et al found, within each age stratum of the NSH, that tinnitus was twice as common in those with a history of occupational exposure to noise (defined as having ever worked in a place for more than six months where the voice had to be raised to be heard) as in unexposed subjects. A strong association has also been reported between tinnitus and sensorineural hearing loss (including noise induced hearing loss). ${ }^{24-26}$ Our findings are consistent with these observations.

In estimating the attributable burden of disease, we standardised for age and sex at the last national census, but not for occupation. As the analysis omitted members of the armed forces (among whom hearing loss was uncommon), and the occupational distribution of the remaining sample was otherwise similar to that at the census, this was considered an adequate means to derive these estimates.

Previous estimates of the frequency of noise induced hearing loss in Britain have come largely from compensation claims under the Department of Social Security's Industrial Injuries Scheme. In 1995/96, 531 new claimants were assessed for benefits, and it was estimated that some 14200 people were receiving compensation payments. ${ }^{3}$ However, this scheme is strongly influenced by awareness and willingness to claim. Moreover, awards are only made for a loss of at least 50 $\mathrm{dB}$ in both ears averaged over the 1,2 , and $3 \mathrm{kHz}$ bands (a quite substantial impairment) in workers employed for at least 10 years in a list of specified noisy occupations. As such, this does not provide a sufficient basis for determining the number of attributable cases of hearing impairment nationally.

Information is also available from two Labour Force Surveys of self reported, work related illness (SWI) carried out by the Health and Safety Executive in 1990 and 1995. In the second of these surveys, ${ }^{4}$ it was estimated that some 140000 adults had deafness caused or made worse by their work (including 31000 who also had tinnitus); and that an additional 10000 adults had work related tinnitus only. The determination of deafness caused by work was based on a review of the answers to several questions which provided evidence of work conditions being the causal link between occupation and deafness ( $J$ Jones, personal communication). An attempt was made to contact respondents' doctors to confirm self reports of illness, and where replies were received, these tended to support the reported diagnosis. In contrast to our own survey, however, cases were only ascertained if they themselves attributed their symptoms to an occupational exposure. As the link with occupation, in a disorder which is insidious, progressive, and an expected concomitant of ageing, may not be apparent to the individual concerned, this is likely to represent a conservative estimate of the attributable burden of hearing loss.

These estimates and our own may also be conservative to the extent that subjects quite often have worse measurable levels of hearing than they admit to, and both surveys focused on overall hearing abilities, rather than deficits in the better ear. Such estimates also depend critically on the definition of hearing impairment, with insurance companies compensating lesser degrees of hearing loss than the State Industrial Injuries Benefit Scheme. ${ }^{27}$ Finally, our own estimate was confined to workers aged 35-64 years, as retired workers were not sampled. A substantial number of retired workers who were not studied may also have noise induced hearing loss.

Direct comparison of our findings with those of the 1995 SWI is hampered by differences in methods, case definition, and the age groups studied (the SWI sampled adults from private households using an interview administered questionnaire). However, our estimates on attributable hearing loss are similar to those of the SWI, while our findings on persistent tinnitus suggest a bigger problem than previously indicated.

Persistent tinnitus and significant difficulties of hearing are quite common, therefore, in Great Britain-especially in men 
in the older working age range, among whom the burden of hearing impairment attributable to noise is substantial. Unfortunately, in some occupations where hearing conservation measures are necessary and important, there is continuing evidence of poor compliance ${ }^{28}$ and of limited provision of audiometric screening. ${ }^{29}$ Our observations highlight the public health impact of occupational exposure to noise and the need for close attention to preventive measures.

\section{ACKNOWLEDGEMENTS}

The Royal College of General Practitioners, the Primary Care Rheumatology Society, HM Armed Forces, and 34 general practices assisted in assembling the mailing lists, and many MRC staff were involved in data collection and processing, especially Ian Bowes and Vanessa Cox. Denise Gould prepared this manuscript. The research was made possible by a grant from the Health and Safety Executive.

\section{Authors' affiliations}

K T Palmer, H E Syddall, B Pannett, D Coggon, MRC Environmental Epidemiology Unit, Community Clinical Sciences, University of Southampton, UK

M J Griffin, Institute of Sound and Vibration Research, University of Southampton

A Davis, MRC Institute of Hearing, University of Nottingham, UK

\section{REFERENCES}

1 Health and Safety Commission. Prevention of damage to hearing from noise at work. Draft proposals for Regulations and Guidance. London: HMSO, 1987

2 Jones JR, Hodgson JT, Osman J. Self-reported working conditions. Health and Safety Executive. London: HMSO, 1995.

3 Health and Safety Commission. Health and safety statistics 1996/7. London: HMSO, 1997.

4 Jones JR, Hodgson JT, Clegg TA, et al. Self-reported work-related illness in 1995. Results from a household survey. Sudbury: HSE Books HMSO, 1998

5 ABI. Occupational disease enquiry 1995. Association of British Insurers, 1996.

6 Palmer K, Coggon D, Griffin M, et al. The development of a self-administered questionnaire to assess exposures to hand-transmitted and whole body vibration and their health effects. J Sound Vib 1998;215:653-86.

7 Palmer KT, Griffin M, Bendall H, et al. The prevalence and pattern of occupational exposure to hand-transmitted vibration in Great Britain findings from a national survey. Occup Environ Med 2000;57:218-28.

8 Iki M, Kurumatani N, Hirata K, et al. Association between vibration-induced white finger and hearing loss in forestry workers. Scand J Work Environ Health 1986;12:365-70.
9 Palmer KT, Coggon D, Syddall H, et al. Occupational exposure to noise and hearing difficulties in Great Britain. CRR 361/2001. Sudbury: HSE Books, 2001

10 Health and Safety Executive. Noise at work. Guidance on regulations. London: HMSO, 1989.

11 Davis AC. The prevalence of hearing impairment and reported hearing disability among adults in Great Britain. Int J Epidemiol 1989;18:911-17.

12 Coles RRA. Epidemiology of tinnitus: (1) Prevalence. J Laryngol Otol 1984;9:7-15.

13 Barone JA, Peters JM, Garabrant DH, et al. Smoking as a risk factor in noise-induced hearing loss. J Occup Med 1987;29:741-5.

14 Virokannas H, Anttonen H. Dose-response relationship between smoking and impairment of hearing acuity in workers exposed to noise. Scandinavian Audiology 1995;24:211-16.

15 StataCorp. Stata Statistical Software: release 5.0. College Station, TX: Stata Corporation, 1997.

16 Zocchetti C, Consonni D, Bertazzi PA. Estimation of prevalence ratios from cross-sectional data. Int J Epidemiol 1995;24:1064-5.

17 Office of Population Censuses and Surveys. Census 1991: Economic activity report. London: HMSO, 1993

18 Efron B, Tibshirani R. An introduction to the bootstrap. New York: Chapman and Hall, 1993.

19 Hinchcliffe R. Prevalence of the common ear, nose, and throat conditions in the adult rural population of Great Britain. A study by direct examination of two random samples. Br J Prev Soc Med $1961 ; 15: 128-40$

20 D'Souza MF, Irwig LM, Trevelyan HT, et al. Deafness in middle age-how big is the problem? J R Coll Gen Pract 1975;25:472-8.

21 Quaranta A, Assennato G, Sallustio V. Epidemiology of hearing problems among adults in Italy. Scand Audiol 1996;25(suppl 42):7-11.

22 Office of Population Censuses and Surveys. General Household Survey: the prevalence of tinnitus, 1981. OPCS Monitor GHS 83/1, Information Branch (Dept M). London: OPCS, 1983.

23 Coles RRA, Davis AC, Haggard MP. Epidemiology of tinnitus. In: Tinnitus (Ciba Foundation symposium 85). London: Pitman Books Ltd, 1981:16-34.

24 Chung DY, Gannon P, Mason K. Factors affecting the prevalence of tinnitus. Audiology 1984;23:441-52.

25 Davis AC. Effects of noise and socio-economic factors on hearing impairment. Proceedings of 4th International Conference on Noise as a Public Health Problem, Torino, July 1983. Maryland: American Speech-Language-Hearing Association, Asha Report, 1983.

26 MacShane DP, Hyde ML, Alberti PW. Tinnitus prevalence in industrial hearing loss compensation claimants. Clin Otololaryngol 1988; 13:323-30

27 Jones CM, Hughes KB. Hearing and vestibular problems. In: Cox RAF Edwards FC, Palmer K, eds. Fitness for work: the medical aspects, 3rd edn. Oxford: Oxford University Press, 2000:190.

28 Lusk SL, Kerr M, Kauffman SA. Use of hearing protection and perceptions of noise exposure and hearing loss among construction workers. Am Ind Hyg Assoc J 1998;59:466-70.

29 Reilly MJ, Rosenman KD, Kalinowski DJ. Occupational noise-induced hearing loss surveillance in Michigan. J Occup Environ Med 1998;40:667-74 